\title{
A cloud infrastructure for scalable computing on population imaging databanks
}

\section{Ignacio Blanquer*}

Institute of Instrumentation for Molecular Imaging (I3M),

Universitat Politècnica de València, Spain

and

GIBI230,

Polytechnic University Hospital La Fe (HUPLF),

Valencia, Spain

Email: iblanque@dsic.upv.es

*Corresponding author

\section{Miguel Caballer}

Institute of Instrumentation for Molecular Imaging (I3M),

Universitat Politècnica de València, Spain

Email: micafer1@upv.es

\section{Luis Martí-Bonmatí}

Biomedical Imaging Research Group (GIBI230) and

Radiology Department,

Hospital Universitario y Politécnico La Fe,

Valencia, Spain

Email: luis.marti@uv.es

\section{Ángel Alberich-Bayarri}

Biomedical Imaging Research Group (GIBI230),

La Fe Health Research Institute, Spain

Email: alberich_ang@gva.es

\section{María de la Iglesia}

Centre of Excellence in Biomedical Image (CEIB),

Regional Ministry of Health in the Valencia Region (CS),

Brain Connectivity Lab. Unidad Mixta FISABIO/CIPF, Spain

Email: delaiglesia_mar@gva.es 


\title{
Jacobo Martínez
}

Valencian Biobank Network,

Foundation for the Promotion of Health and

Biomedical Research of Valencian Region (FISABIO),

Valencia, Spain

Email: martinez_jac@gva.es

\begin{abstract}
This article describes the software architecture designed to cope with the computing demand of research usage of complex data from the imaging biobank of the Regional Ministry of Health in the Valencia Region (CS). It proposes the use of self-configured virtual clusters on top of on-premise and public cloud infrastructures. It uses a model based on recipes and autoconfiguration to deploy virtual elastic clusters that adjust themselves to the actual workload of the study, therefore reducing operating costs and preventing the need of up-front investments both at the level of the imaging biobank or the final user. All the software used is released under open-source licenses.
\end{abstract}

Keywords: imaging population; cloud computing; virtual infrastructures; biobanks.

Reference to this paper should be made as follows: Blanquer, I., Caballer, M., Martí-Bonmatí, L., Alberich-Bayarri, Á., de la Iglesia, M. and Martínez, J. (2015) 'A cloud infrastructure for scalable computing on population imaging databanks', Int. J. Image Mining, Vol. 1, Nos. 2/3, pp.175-188.

Biographical notes: Ignacio Blanquer has been a Professor at the DSIC Department of the Universitat Politècnica de València since 1999 and GRyCAP member since 1993. He is currently the Head of this research group. He has authored and coauthored more than 70 papers in national and international journals. His research interests include grid and cloud systems, medical image instrumentation and high performance computation.

Miguel Caballer received his BSc, MSc and $\mathrm{PhD}$ in Computer Science from the Universidad Politécnica de Valencia (UPV), Spain in 2000, 2012 and 2014, respectively. He is part of the grid and high performance computing group of UPV since 2001. He has participated in different research projects about the application of grid and cloud computing techniques to several areas of engineering. His other fields of interest include green computing.

Luis Martí-Bonmatí is a Professor of Radiology. He has been chairing the Radiology Department and the Biomedical Imaging Research Group (GIBI230) at La Fe University and Polithecnical Hospital since 2011. He is the President of the European Society of Gastrointestinal and Abdominal Radiology and Secretary General of the International Society of Radiology. He has authored and coauthored more than 300 papers in national and international journals. His main research interests include radiology, imaging biomarkers, imaging processing, abdominal imaging and magnetic resonance imaging.

Ángel Alberich-Bayarri is the Scientific-Technical Director of the Biomedical Imaging Research Group of La Fe University and Polytechnics Hospital. He is also the founder of the start-up QUIBIM. He has authored and co-authored more than 40 papers in national and international journals. His research interests include quantitative radiology, image processing techniques, imaging biomarkers and imaging biobanks. 
María de la Iglesia is a civil servant in the Valencia Regional Ministry of Health. Currently, she is the Head of the Brain Connectivity Lab. in the Department of Neurological Impairment at Príncipe Felipe Research Centre of Valencia (joint unit FISABIO-CIPF). Also she is part of the Interim Board in Euro-BioImaging as scientific delegate by Spain in the field of medical imaging since 2014 in the expression of interest 'population imaging'. She has authored and coauthored more than 20 papers in national and international journals. Her research interest includes methodologies with high performance computational image used for the analysis of functional and structural brain imaging and the development of statistical, data mining methods and bioinformatics tools to understand and analyse information of population imaging.

Jacobo Martínez received his BSc in Chemistry from the Valencia University in 1995. He received his post graduate diploma in Biochemistry and Clinical Pathologies from the Valencia University in 1996. He received his Master degree in Biotechnology from the International Business School ALITER in 2003. He has worked for different biotech companies dealing with business development issues. Since 2008, he has been the Manager of a Population-Based Biobank, the IBSP-CV Biobank, and Head of the Valencian Biobank Network. He has been a principal investigator of several national funded projects. He is also a member of the General Council of the Spanish National Biobank Network.

\section{Introduction}

Population imaging and large datasets of medical images are a clear need. To further develop personalised medicine, quantitative medical imaging needs to clinically assess value, reproducibility and universality. By ensuring the development and implementation of signal modelling and image features extraction strategies, quantitative imaging will help to prevent or effectively treat diseases. Virtual biobanks, as repositories of digital information, have increased the opportunities for sharing, federating and exploiting large datasets of patients' information. Medical imaging biobanks can be considered as virtual biobanks. They recently emerged for advancing on the study of diseases, identification of early disease biomarkers and surrogates, and development of population studies. These imaging biobanks will evaluate the impact of new quantitative biomarkers, both resolved in space and time on parametric and nosologic images, on early disease diagnosis, disease phenotyping, disease grading, targeting therapies and evaluation of disease response to treatment.

Imaging and engineering technologies support retrospective and prospective epidemiological studies on the population level. Specific markers obtained by large scale image processing of pre-symptomatic diseases might be used to evaluate different biological and pathological abnormalities, new disease phenotyping and early identification of people at risk. 
The European strategy forum on research infrastructures (ESFRI) mission leads to the better use and development of research infrastructures, both at the European and international levels. Within this initiative, Euro-Biolmaging is a European project establishing a distributed set of open access nodes. Although many new imaging technologies and quantitative initiatives are arising, such as multiparametric approaches, their application to clinical practice is limited, most remaining on the research field. One important reason for this limitation is the need for standardised platforms in which different procedures, protocols and techniques can be evaluated on suitable images. Such platforms should be able to solve the strong requirements on data computing and storage. Our Euro-BioImaging node main objective is to set up an innovative platform for the execution of multicentre clinical trials and research projects based on medical imaging and imaging biomarkers. This advanced imaging platform will enable to test and validate state-of-the-art image analysis techniques, increase quality and accuracy of clinical trials through the use of advanced quantitative imaging techniques, promote new visualisation tools and, finally, reduce overall costs.

Similarly, in the last years several collaborative environments have been developed together with powerful tools for the exchange of knowledge among medical institutions, improving diagnosis and research. Most of them primary rely on medical images, and they use grid and cloud computing infrastructures to organise the access and the use of the various services offered by them. Such collaborative environments include the biomedical informatics research network (BIRN) from Grethe (2005), the e-science platform for researchers of Alzheimer's disease neuGRID (Redolfi et al., 2009), its recent successor N4U or the integrated healthcare platform for European paediatrics health-e-child from Marcus et al. (2007). Representative examples of medical imaging applications exclusively using Cloud computing include the work of Silva et al. (2012) for the storage of medical studies using public cloud providers, and a Windows Azure-based DICOM server from Teng et al. (2010). Those platforms offer fixed environments which are infrastructure-dependent.

\section{Requirements}

According to the European Society of Radiology (ESR, 2014) working group on imaging biobanks definition, imaging biobanks are defined as organised databases of medical images and associated imaging biomarkers (radiology and beyond) shared among multiple researchers. The definition already considers the interaction of specialists from different disciplines with the platform.

\subsection{Use cases as defined by applications of the platform}

Therefore, the needs to be covered by this advanced imaging platform are directly related to the most relevant use cases for medical imaging and that are considered in its design, consisting on: clinical research, development of new imaging biomarkers, imaging-based clinical trials and biobanks. 
The platform will be an environment for clinical research, where the users will have the capability of choosing the data they want to statistically process for their study, including not only images but also associated imaging biomarkers and meta-data in a way of customised research based on imaging. The data in the platform will be properly anonymised if not having written informed consent and de-identified in the case of having a written informed consent (e.g., those coming from clinical trials). The data will cover the entire spectrum of human disease and provide a platform where state-of-the-art image analysis algorithms can be evaluated on own data.

This population-based imaging biobank will be used also to fill the gap between research and clinical application in quantitative imaging and imaging biomarkers. Nowadays, there is a need to validate either existing or new quantitative imaging methods. As an example, several imaging biomarkers exist as candidates to early evaluate treatment response in oncology, but large cohorts are required in order to extract robust conclusions and relevant evidence for clinical practice and clinical trials. Image processing scientists will have the capability of uploading and sharing their own algorithms and methods with other researchers, to be tested with the studies in our platform. The platform will also ease the development and validation of novel image analysis techniques and their comparison with implementations of other research groups applied to the same cohorts.

The number of clinical trials based on imaging is progressively increasing, due to the better technological capabilities of the different modalities that allow for more precise and accurate diagnosis. However, the use of quantitative imaging in clinical trials is still an important challenge, since there is a lack in the standardisation of image analysis procedures. Our platform will include image analysis algorithms following criteria of the scientific community and novel standardisation initiatives like the quantitative imaging biomarkers alliance (QIBA).

Finally, the mechanisms for the connection of this imaging platform with existing biorepositories will be also considered. A biorepository is a biological material repository that collects, processes, stores, and distributes biospecimens to support future scientific investigation. Although a biorepository can contain or manage specimens from animals, including humans, and many other living organisms, in some discussions, such as in ISBER (2001), the term is reserved for human specimens.

Since the late 1990s, biorepositories have became an important resource in medical research, supporting many types of contemporary research like genomics and personalised medicine. For example, many diseases are associated with single-nucleotide polymorphisms, and performing genome-wide association studies using large collections of samples which represent tens or hundreds of thousands of individuals can help to identify disease biomarkers as stated by Greely (2007).

Hewitt (2011) and many other researchers have identified biobanking as a key area for infrastructure development in order to promote drug discovery and drug development. These data are currently available in local databases and the mechanisms of connectivity and access to them will be addressed.

The connection of biorepositories and medical image databanks enable the guidance to the development of advanced imaging surrogates that could lead to early diagnosis and to anticipate the most effective therapy. 


\subsection{Use cases as defined by the types of interaction}

According to the way the resources will be used, three use cases are considered:

- Interactive usage. A project requires to analyse and process manually a set of images from the case studies selected. A single virtual machine (VM) - with a specific virtual hardware configuration - is needed. The VM is properly configured with a set of tools and Interactive access is required.

- Batch. A project requires applying a straight but computationally intensive processing on a wide range of images. Unattended execution is possible and desirable. Once the parameters are fixed, a simple combination of programs is executed over the whole bunch of images. It will require multiple VMs and a local resource management system (such as Torque, PBS, LSF, OGE, etc.)

- Pipeline. A project requires applying a complex processing on a set of images Requiring multiple steps and synchronisations with different requirements (for example, it requires merging the results from previous steps). It will require multiple VMs and a pipeline manager able to work with the infrastructure.

\subsection{Image processing requirements}

The platform will integrate data with a high degree of heterogeneity, like medical images with all the interpretations of DICOM(http://dicom.nema.org) standards from the different manufacturers, parametric maps of imaging biomarkers, 3D reconstructions of anatomy, source codes of image processing algorithms, and associated clinical data and variables. The main data types managed by the platform will be plain DICOM files (one DICOM file per image) and also NIFTii and analyse formats (*.nii and *.hdr $/ *$.img file extensions, respectively), that can handle entire volumes in a single file and are widely extended among medical image processing scientists community.

For data conversion, the MRIcron (McCausland Center for Brain Imaging, Columbia SC, USA) package will be included in the platform. For data analysis the platform will include a broad range of tools, such as the FSL (FMRIB Software Library, Analysis Group, Oxford, UK) and SPM (Wellcome Trust Center for Neuroimaging, University College London, London, UK) packages that are specially suited for neuroimaging analysis, and a connector for Python scripts, in order to allow the evaluation of algorithms developed by the users of the platform.

\subsection{Pipelining and processing}

A pipeline-based architecture will be used for the development of quantitative imaging procedures. Image analysis methods will be classified in those used for quality control data (i.e., signal noise ratio plot), data pre-processing (i.e., segmentation, filtering, interpolation), data analysis (i.e., brain volumes quantification, T2 mapping, perfusion analysis, lung emphysema quantification) and data measurement (i.e., histogram based analysis, multivariate statistical analysis). Rules will be defined for the interconnection of the different types of processing modules for pipeline creation. 


\section{Materials}

According to the above requirements, the management of individual subprojects has to deal with the following points:

- Each subproject will have specific requirements (in terms of resources and applications) that have to be fulfilled individually.

- Multi-tenancy of the computing infrastructure does not need that data can be accessible by different projects. Data, even anonymised, and produced results must be protected from the access of unauthorised users.

- Projects may require resources or have external resources available, and the applications should work seamlessly.

In this scenario, we define a software architecture that instantiates virtual appliances customised for each subproject. A virtual appliance is a set of fully configured VMs that run coordinately.

In all cases, the infrastructure management service will provide fully-configured virtual appliances. The software required will be described as auto configuration recipes. In this part we envisage two types of software components:

- Commodity components. The Valencia EuBio node (BIMCV) offers a range of widely used open-source processing programs and toolkits such as FSL, SPM, mricron. Those components are available in a market place as a set of customised recipes that are used by the infrastructure manager (IM) for setting up the resources. Those recipes include the minimum virtual hardware requirements, which can be overwritten in each specific project if needed.

- Custom components. Each project can include their own components to the Virtual Appliances. Users are encouraged to prepare configuration recipes to facilitate autoscaling automatic configuration. Nevertheless, users can manually administer their own virtual resources adding more components. However, this last feature is limited to the configuration running on end-users' premises or public clouds, to reduce the risk of running malicious software on BIMCV's premises.

Therefore, our platform defines the following services:

- a catalogue of images of preconfigured VMs including plain images for further contextualisation and complete stacks

- a recipe registry with automatic installation instructions for a wide range of processing components

- an IM for the deployment and reconfiguration of virtual appliances

- a service for the automatic management of elastic clusters based on the workload

- a workflow manager able to deal with elastic hybrid pipelines

- a computing infrastructure based on a cloud on-premise

- a final user application that enables requesting the virtual appliances and deploying them on the desired infrastructure. 
The previous services are described in the following sub-sections.

\subsection{Virtual resource management catalogues}

The widespread usage of virtualisation requires creating virtual machine images (VMIs), which encapsulate the required hardware and software configuration required for the applications. Different applications impose different software and hardware requirements, which leads to a massive creation of VMIs which must be properly indexed in order to leverage both their reuse and sharing. For that, Carrión et al. (2010) proposes a catalogue and repository of VMIs that enables the users (and/or cloud administrators) to index and store the VMIs together with the appropriate metadata that describes their hardware and software features. The VMRC system can be deployed within a private cloud to store the different VMIs required to provision the services. It can also index VMIs stored within other cloud providers. In addition, it can be employed to federate different private clouds so that a single VMRC entity can provide the images for different clouds. The catalogue itself is hypervisor-agnostic and it uses the open virtual format (OVF) specification. The catalogue is extended with a repository of ansible-based recipes (Hochstein, 2014), which enables setting-up not only the proper basic VMI, but additionally the software to be configured in the virtual appliance. In this way, software configurations are described as a set of modules to be included in the final set-up.

\subsection{Automatic configuration of virtual appliances}

Cloud infrastructures can be used to address the computational needs in multiple domains, including scientific applications. However, the use of public or on-premises infrastructure as a service (IaaS) clouds require non-trivial system administration skills. If a single VM is needed, custom configurations available in public clouds may be sufficient to meet users' requirements. When multiple VMs are needed, users have to configure shared directories, batch queues, administer users and customise software. For that, the IM, firstly presented in Caballer et al. (2014) and de Alfonso et al. (2011), is a tool that ease the access of IaaS clouds by automating the selection of the proper VMI, and the further deployment, configuration, software installation, monitoring and update of virtual appliances.

IM provides an abstraction layer to enable the interoperability with different IaaS clouds. This layer has been designed using a plug-in scheme that currently provides plug-ins for: OpenNebula, OCCI, Amazon EC2, Google Cloud, Docker, OpenStack and libvirt. This set of plug-ins enables the access to a large number of cloud deployments and virtualisation platforms, thus enabling the user to start using a simple virtualisation system and then transparently migrating to the cloud. It integrates ansible as the contextualisation system to enable the installation and configuration of all the user required applications providing the user with a fully functional infrastructure. Despite that multiple cloud providers offer services for the deployment of software configurations (e.g., HEAT from OpenStack or the software management and catalogue service of OpenNebula), IM recipes are compatible to all the platforms supported via plug-ins. 
Figure 1 Description of the configuration of a virtual appliance (see online version for colours)
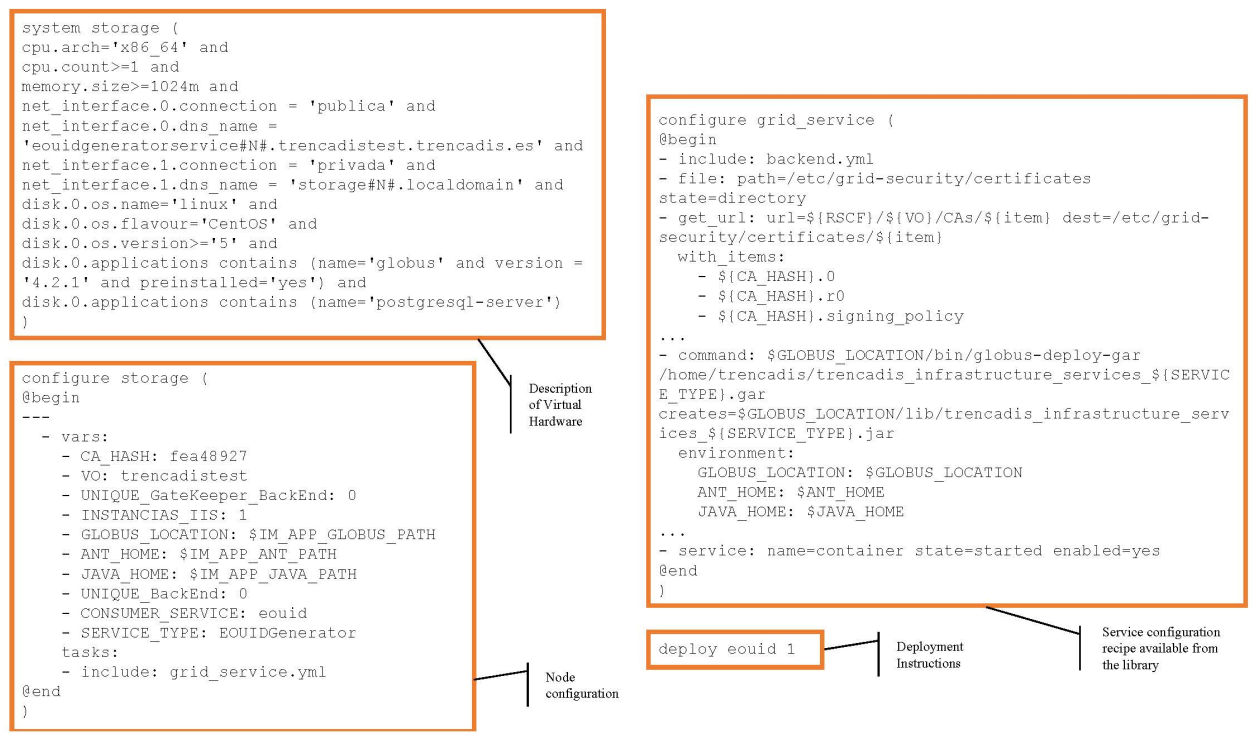

Note: Example of a storage resources.

Figure 1 shows two examples of configuration recipes. The one in the left describes the structure of a configuration of a medical imaging storage node, which includes four parts:

- the description of the virtual hardware

- the deployment of the node

- the common configuration recipes

- the instantiation of variables specific of this node.

Despite that on-the-fly configuration introduces an overhead, this overhead is only compulsory for creating the virtual images for the first time. VMs can be preserved without requiring being active along the life-time of the project. Snapshots of the VMs can be performed and registered in a catalogue for longer-term hybernation. An interesting alternative, which is also supported in IM, is the use of docker containers. This will enable a binary-compatibility level with cloud providers (including public clouds like the container service of Amazon). IM can instantiate those containers and they can be registered in a catalogue for further reuse.

\subsection{Elastic virtual appliances}

Elastic cloud computing cluster (EC3) (Caballer et al., 2013) is a tool to create elastic virtual clusters on top of IaaS providers, either public (such as Amazon web services) or on-premise (such as OpenNebula and OpenStack). We offer recipes to deploy TORQUE (optionally with MAUI), SLURM and SGE clusters that can be self-managed with CLUES: it starts with a front-end node and working nodes will be dynamically deployed and provisioned to fit increasing load (number of jobs at the LRMS). Working nodes will be undeployed when they are idle. This introduces a cost-efficient approach for 
cluster-based computing. Clusters can be complemented in a hybrid model by the creation of VPNs that extend a virtual cluster (Amanda et al., 2014) deployed in on-premise infrastructures and complemented with additional resources from a public cloud provider. This model is feasible when the inter-node communication and the access to a shared file system is small in comparison with the computational cost.

\subsection{Infrastructure}

The platform is designed to be 'cloud-agnostic', being able to work with multiple infrastructures. In the present time, three kinds of infrastructures are considered: Local infrastructure of BIM-CV. This infrastructure is provided by the UPV and the CIPF, with a total capacity of 100 virtual instances. It is currently provisioned using OpenNebula.

External infrastructures. In this point we consider both public infrastructures such as Amazon AWS and research infrastructures such as EGI Federated Clouds. The framework supports already both AWS EC2 (Amazon proprietary API) and r-OCCI (the standard interface for managing cloud infrastructures, supported by EGI federated clouds).We use end-users' own credentials for the former and robot certificates for the latter. End-users' own infrastructure. The infrastructure management service is able to deploy the VMs directly on an end-user's on-premise cloud (supporting both ONE and OpenStack, or even libvirt-based resources).

The virtual infrastructures will expose a XNAT (eXtensible Neuroimaging Archive Toolkit, http://xnat.org/) instance. Marcus et al. (2007) is an open source platform designed to facilitate the management of image sets and associated data (assessments, reconstructions and any other information). Initially it is designed to work with neuro imaging but the open data model and customisable XML-based technologies allow to adapt the platform for any type of bioimaging. XNAT follows a three-tier architecture that includes a data file, an user interface and a middleware engine. The data file can be incorporated into the platform through different ways, such as XML files, web forms, DICOM transfers from image capture devices or image viewers like Oxiris and so on. Among its most important features are the personalised safe access to information, quality control processes of data and image information, classification and storage of data, ability to run custom searches, communication with bioimaging generating systems, programmability of process flows using scripts (pipelines), the incorporation of intermediate results and conclusions to the study, etc. All these features make XNAT an ideal platform for the management of clinical trials.

\section{Methods}

The general procedure BIMCV addresses goes through the following steps:

- A proposal of a project requesting data is prepared using a set of standardised forms. These forms include the needs for data, software and computing resources. The proposals are presented in a two-stage model. In the first stage, the proposal is evaluated by a technical committee, that checks the viability of the request, the 
availability of data and the ethical compliance. After a positive answer, proposers must present a more detailed version of the project which is evaluated by a scientific committee.

- The scientific committee revise the project and proposes a final assignment of resources (if needed). Those resources are defined as a credit in hours that can be spent on BIMCV's computing infrastructures.

- Data is extracted and anonymised from the regional PACS and manually verified by an expert, which complements the data with the necessary clinical information. The result is a dataset that is copied into a virtual disk volume. This virtual disk volume will be attached to the virtual infrastructure.

- A technical expert defines the proper configuration of the virtual appliance. A recipe is created with the components selected and the features of the virtual hardware. This recipe will be used to instantiate and reconfigure the virtual appliance.

- Deploy and monitor the virtual appliance in the infrastructure proposed. In case of using BIMCV's infrastructure, a real-time accounting is provided to check the availability of resources.

Next section describes the deployment architecture.

\subsection{Architecture deployment}

A virtual cluster is assigned to each project. This virtual cluster uses a base Virtual Image from the virtual machine resource catalogue (VMRC) and contextualises it with two types of instances:

- A master node that manages the virtual cluster through a batch queue (using torque). This master node is also instrumented with the automatic elasticity component (Caballer et al., 2014). This master node will interact with the IM to deploy or undeploy a number of working nodes.

- A working node image that will be used for processing the individual batch jobs that a pipeline generates. This stateless working nodes will be instantiated by the master node.

Figure 2 shows the deployment of a project-specific virtual appliance. The data resulting from a query to the regional PACS is prepared in a virtual disk volume, which will be part of the Virtual Appliance. The autoconfiguration accesses the recipes database and using the project specification configures two VM images with the front-end and the working nodes. Those images are instantiated in the desired infrastructure. The service is infrastructure agnostic, supporting OCCI, EC2, ONE, OSTACK and libvirt interfaces currently, so it can work with public clouds, the on-premise cloud of BIM-CV or end-users' own resources. End-users can connect through the front/end node, which provides the desired interface according to the components and recipes available in the marketplace. 
Figure 2 Deployment of a project-specific virtual appliance

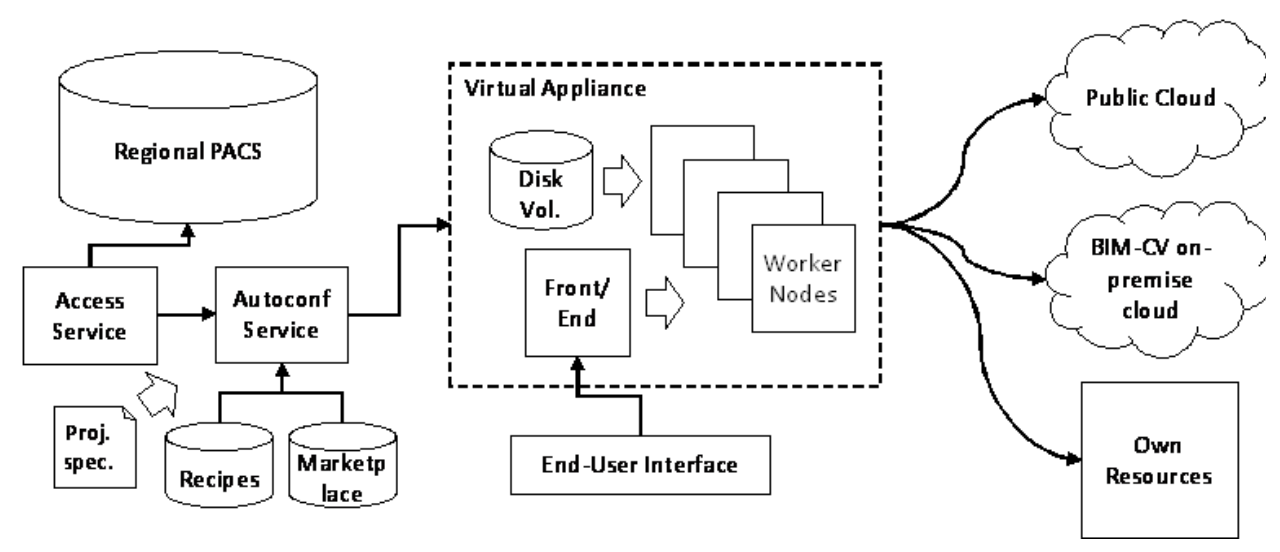

\section{Conclusions and further work}

The need of computing platforms in population imaging analysis is clearly justified by the huge demand of resources that requires processing large cohorts of complex images. In this sense, cloud computing has emerged as a solution to feed this request. However, the direct usage of public cloud infrastructrues undercover several challenges that are addressed by the work presented.

Each public cloud provider has its own (incompatible) way to represent VMIs. Migration from one provider to another is not automatic since it will require converting VMIs and reimplement the elasticity management. Moreover, the configuration of virtual clusters require system administration skills. Vendor lock-in, lack of control, IaaS management and security refrain Medical Imaging researchers to adopt cloud computing in a wider scale. In this sense, the use of recipes and on-the both eases infrastructure deployment and isolates from vendor's VMI particularities. Moreover, infrastructures can be even deployed on local on-premise infrastructures. Finally, recipes can be exposed in a library and re-used by non-expert users to deploy complex configurations.

The deployment on the fly increases the delay on the availability of resources. However, this delay is only suffered in the first instantiation of the virtual infrastructure and Caballer et al. (2014) shows reasonable re-configuration delays when new resources are added or removed from the infrastructure. The scenario of BIM-CV focuses on long-lasting infrastructures that can be elastically increased or decreased.

Elasticity is a requirement for budget contention (either real or virtual credits) in any cloud infrastructure. Elasticity should be automatic and depending on the workload. The use of pipelines facilitates the implementation of IaaS elasticity rules based on the length of the processing queue. Results in Caballer et al. (2013) show very good performance, especially in coordination with workflow engines.

Finally, the deployment of individual virtual infrastructures is a better approach than sharing a physical infrastructure even in the case that computing and software requirements of the subprojects concur. Multitenancy at the level of the infrastructure enables isolating users of different subprojects even more, and reduces the risks of 
crashes, infrastructure overloading and maintenance failures with respect to the traditional mechanisms based on quotas.

Therefore, the model of virtual elastic infrastructure fits well the requirements both of the users and the infrastructure providers.

Euro-BioImaging is currently in the intermim phase, and planning to start prototyping services during 2015. The BIMCV has identified preliminary sub-projects to be used as prototypes for the infrastructure proposed in the article. Currently, the platform is implemented at the level of the provisioning services. Current work is being focused on the access service to data. In this piloting phase, BIMCV will run standard medical-imaging benchmarks and populate the software repository.

In a second stage, BIMCV is planning to upgrade the software architecture with the support of vertical elasticity and the use of containers. Vertical elasticity will enable running memory-demanding applications in a workflow without requiring the pre-reservation of large-scale instances.

\section{References}

Caballer, M., Blanquer, I., Moltó, G. and de Alfonso, C. (2014) 'Dynamic management of virtual infrastructures', Journal of Grid Computing, doi: 10.1007/s10723-014-9296-5.

Caballer, M., de Alfonso, C., Alvarruiz, F. and Moltó, G. (2013) 'EC3: Elastic Cloud Computing Cluster', J. Comput. Syst. Sci., December, Vol. 79, No. 8, pp.1341-1351.

Caballer, M., de Alfonso, C., Moltó, G., Romero, E., Blanquer, I. and García, A. (2014) 'CodeCloud: a platform to enable execution of programming models on the clouds', Journal of Systems and Software, July, Vol. 93, pp.187-198.

Calatrava, A., Moltó, G., Caballer, M. and De Alfonso, C. (2014) 'Virtual hybrid elastic clusters in the cloud', 8th Iberian Grid Infrastructure Conference (IberGrid 2014).

Carrión, J.V., Moltó, G., De Alfonso, C., Caballer, M. and Hernandez, V. (2010) 'A generic catalog and repository service for virtual machine images', 2nd International ICST Conference on Cloud Computing (CloudComp).

de Alfonso, C., Caballer, M., Alvarruiz, F. and Hernández, V. (2013) 'An energy management system for cluster infrastructures', Computers and Electrical Engineering, Vol. 39, No. 8, pp.2579-2590, ISSN 0045-7906, 10.1016/j.compeleceng.2013.05.004.

de Alfonso, C., Caballer, M., Alvarruiz, F. and Moltó, G. (2013) 'An economic and energy-aware analysis of the viability of outsourcing cluster computing to the cloud', Futur. Gener. Comput. Syst. (International J. Grid Comput. eScience), March, Vol. 29, No. 3, pp.704-712.

de Alfonso, C., Caballer, M., Alvarruiz, F., Moltó, G. and Hernández, V. (2011) 'Infrastructure deployment over the cloud', 3rd IEEE International Conference on Cloud Computing Technology and Science (CloudCom), pp.517-521, 10.1109/CloudCom.2011.77.

ESR Working Group on Imaging Biobanks (2014) Mission Statements, ESR Working Group on Imaging Biobanks [online] http://www.myesr.org/cms/website.php?id=/en/research/esr_wg _on_imaging_biobanks/esr.htm (accessed 02/01/2015).

Freund, J., Comaniciu, D., Ioannis, Y., Liu, P., McClatchey, R., Morley-Fletcher, E., Pennec, X., Pongiglione, G. and Xiang, Z. (2006) 'Health-e-child: an integrated biomedical platform for grid-based paediatric applications', Stud. Health Technol. Inform., Vol. 120, pp.259-270.

Greely, H.T. (2007) 'The uneasy ethical and legal underpinnings of large-scale genomic biobanks', Annual Review of Genomics and Human Genetics, Vol. 8, pp.343-364. 
Grethe, J.S., Baru, C., Gupta, A., James, M., Ludaescher, B., Martone, M.E., Papadopoulos, P.M., Peltier, S.T., Rajasekar, A., Santini, S., Zaslavsky, I.N. and Ellisman, M.H. (2005) 'Biomedical informatics research network: building a national collaboratory to hasten the derivation of new understanding and treatment of disease', Stud. Health Technol. Inform., Vol. 112, pp.100-109.

Hewitt, R.E. (2011) 'Biobanking: the foundation of personalized medicine', Current Opinion in Oncology, Vol. 23, No. 1, pp.112-119.

Hochstein, L. (2014) Ansible: Up and Running, Automating Configuration Management and Deployment the Easy Way, ISBN: 978-1-4919-1532-5, O'Reilly Media, USA.

International Society for Biological and Environmental Repositories (ISBER) Newsletter (2001) Vol. 1, No. 1.

Marcus, D.S., Olsen, T., Ramaratnam, M. and Buckner, R.L. (2007) 'The extensible neuroimaging archive toolkit (XNAT): an informatics platform for managing, exploring, and sharing neuroimaging data', Neuroinformatics, Vol. 5, No. 1, p.1134.

Myneni, S. and Patel, V.L. (2010) 'Organization of biomedical data for collaborative scientific research: a research information management system', International Journal of Information Management, Vol. 30, No. 3, pp.256-264.

Redolfi, A., McClatchey, R., Anjum, A., Zijdenbos, A., Manset, D., Barkhof, F., Spenger, C., Legré, Y., Wahlund, L.O., Pietro, C.B.d.S. and Frisoni, G. (2009) 'Grid infrastructures for computational neuroscience: the neuGRID example', FutureNeurol., Vol. 4, No. 6, pp.703-722, doi:10.2217/fnl.09.53.

Silva, L., Costa, C. and Oliveira, J. (2012) 'APACS archive architecture supported on cloud services', Int. J. Comput. Assist. Radiol. Surg., Vol. 7, No. 3, pp.349-358, doi:10.1007/s11548-011-0625-x.

Teng, C.C., Mitchell, J., Walker, C., Swan, A., Davila, C., Howard, D. and Needham, T. (2010) 'A medical image archive solution in the cloud', in IEEE International Conference on Software Engineering and Service Sciences (ICSESS), pp.431-434, doi:10.1109/ICSESS.2010.5552343. 